\title{
Capturing tacit knowledge: A case of traditional doctors in Mozambique
}

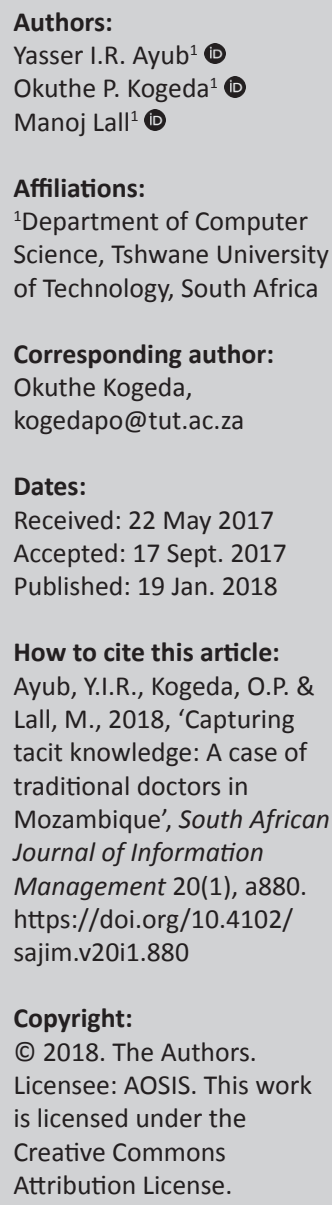

Background: The indigenous healers or traditional doctors play a significant role in the healthcare provisioning in Africa. Although traditional healing has been in practice for several centuries, very little has been achieved in terms of harvesting the wealth of tacit knowledge possessed by these practitioners for future references.

Objective: The objectives of this article were twofold. The first objective was to capture the tacit knowledge possessed by traditional healers in Mozambique. The second objective was to enhance preservation of tacit knowledge for sharing and training of other traditional healers.

Method: The cognitive semiotic model was used as a basis for capturing the tacit knowledge. Qualitative and quantitative data were collected by using interviews and questionnaires were applied during systems requirements elucidation, designing the user interface and in building the knowledge repository. In the requirement elucidation phase, 13 traditional doctors were interviewed. Besides text and voice recordings, video recordings and photos were used in capturing the tacit knowledge. The usability of the system was tested by using the heuristic evaluation technique.

Results: The findings revealed that the system developed could be used for capturing the tacit knowledge and storing it in an explicit form. The usability tests indicated that the prototype developed served its purpose to a great extent.

Conclusion: The study concludes that cognitive semiotic model is a suitable tool for capturing tacit knowledge of traditional doctors in Mozambique. The results of the usability evaluation confirm that the system developed supports information sharing and ease of use. It is observed that the developed application could support users with little formal education. However, some basic computer literacy training would assist in maximising the benefits of the system.

\section{Introduction}

Today, there is very little doubt that one of the most important assets of any organisation is its knowledge and knowledge sharing ability (Geisler \& Wickramasinghe 2015). Knowledge management and its techniques are considered to be important proponents of knowledge sharing and knowledge flow between organisations and individuals. Knowledge is broadly categorised as explicit knowledge and tacit knowledge. Explicit knowledge can be easily codified and shared asynchronously. It is formal, written down and documented knowledge making it relatively easy to share, while tacit knowledge is informal and resides in our minds as mental models, personal experiences and know-how (Polanyi 1966, 2015; Walker 2017). Essentially, tacit knowledge contains two components - a technical component that reflects the know-how of professional activities, and a cognitive component that reflects mental models, beliefs and perceptions as a result of many performed similar actions (Bratianu \& Orzea 2010). Tacit knowledge is often accumulated through a lifetime of experience, experimentation, perception and learning by doing; thus capturing such knowledge is a lot more difficult (Grover \& Froese 2016; Howells 2002; Polanyi 1983).

Although there is an existence of a knowledge base for explicit knowledge, the same is not true for tacit knowledge. The value of harvesting the tacit knowledge held by the traditional doctors is vast and presents new frontiers in the field of knowledge management. However, there is not much work conducted in harvesting of tacit knowledge from traditional doctors (Gibert-Tisseuil 1997). To alleviate this problem, the World Health Organization has created strategies and guidelines for the study and practice of medicinal usage of plants indigenous to Mozambique (Bruschi et al. 2011). 
Mozambique is a country located in the south-east of Africa surrounded by the Indian Ocean to the east (see Figure 1). According to the report released by World Health Organization (WHO 2004) only $40 \%$ of the inhabitants of Mozambique have access to the public health system, and the remainder of the population continues to rely on tacit knowledge of the traditional doctors. In an attempt to preserve and promote sharing of this knowledge, an association of traditional healers was founded in 1990, called AMETRAMO (Associação de prática de Medicina Tradicional de Moçambique). In 2004, national policy on traditional medicine, which included strategies for its development, was established (Misau 2004).

The health department of Mozambique often conducts seminars to facilitate the interchange of knowledge between traditional doctors and modern doctors. Topics related to diagnosis, prevention and treatment of epidemic diseases are discussed. Unfortunately, not all traditional doctors are willing to participate in this exchange of knowledge due to factors such as lack of time and trust and fear that divulging their knowledge would result in a loss of competitive advantage.

The three main categories of traditional health services in Mozambique are: herbalists (inyanga in isiZulu, herbalista in Portuguese), diviners (sangoma in isiZulu, curandeiro in Portuguese) and prophets (profetas in Portuguese).

\section{Herbalists}

They are traditional doctors relying on the use of various plants for healing of common ailments. They predominantly focus on the preparation and administration of traditional medicines derived from various parts of plants (Bruschi et al. 2011).

\section{Diviners}

In many cultures of Mozambique, diseases are believed to be linked to social and spiritual factors (Lechner 2009). They believe that many sicknesses are the result of loss of protection from their ancestors because of having ignored certain rituals or having behaved immorally. An individual seeking spiritual help undergoes a ritual locally known as Fembar. Diviners are known to be traditional doctors dedicated to healing various diseases by using such rituals (Bruschi et al. 2011).

\section{Prophets}

With an increase in a number of churches in Mozambique, many people are leaving behind their traditional animist beliefs and are drawn to churches to resolve problems related to ill health and misfortunes (Pfeiffer et al. 2007). Prophets are people with strong faith and believe that sicknesses are caused by evil spirits within a patient. Treatments usually require holy water and recitation of a holy book (Hey 2004; Pfeiffer 2005). Most of these prophets have little knowledge of the properties or benefits of traditional plants, but rely on the power of prayer and faith (Alavi \& Leidner 1999).

As a vast number of people in Mozambique still rely on traditional doctors for their well-being, the services offered by the traditional doctors are vital. Because the knowledge possessed by these traditional doctors is mostly in the form of personal experiences, know-how, insight and mental models, the harvesting and preservation of such knowledge are crucial. These knowledges are mostly abstract and human dependent and therefore more difficult to codify and share (Burnett, Macafee \& Williams 2017; Nonaka 2008; Nonaka \& Takeuchi 1995; Walker 2017). This research focuses on the important issue of enhancing the sharing of the tacit knowledge possessed by traditional healers of Mozambique. This objective is achieved by applying mechanisms to convert the tacit knowledge into an explicit form. The use of Information Technology (IT) as an enabler for knowledge sharing and preservation is well documented (Johannessen, Olaisen \& Olsen 2001; Neches et al. 1991; Presbitero et al. 2017; Soto-Acosta, Popa \& Palacios-Marqués 2017). Therefore, we make extensive use of IT in harvesting, preserving and sharing the tacit indigenous knowledge of the traditional doctors in Mozambique.

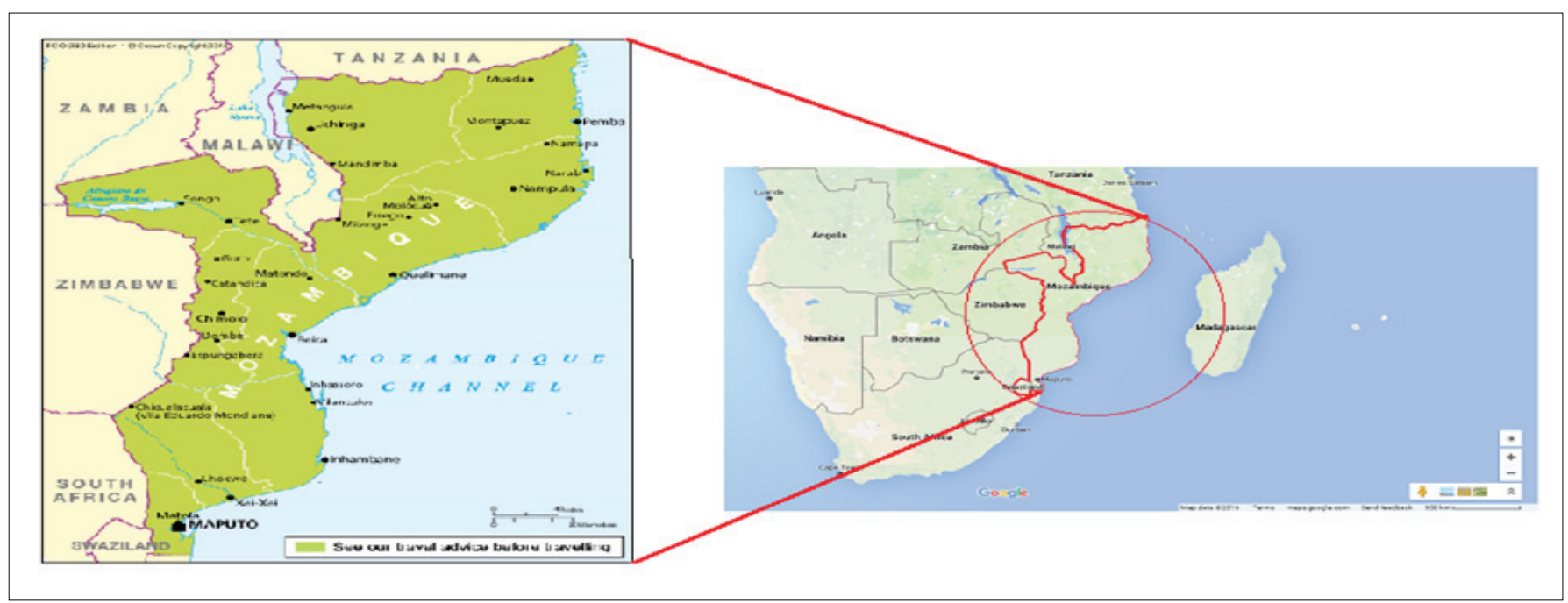

Source: Googlemaps, 2016, Map of southern Africa, viewed 23 April 2016, from https://www.google.co.za/maps/@-20.0347085,27.132872,4.65z?hl=en FIGURE 1: Map of southern Africa. 
The rest of the article is structured as follows: Firstly, the theoretical background and literature review are presented in Section 2. Next, the research methodology used in this research is explained in Section 3. This is followed by results and discussion in Section 4, and lastly, the summary, conclusions and future work are provided in Section 5 .

\section{Theoretical background and literature review}

Knowledge is considered to be a vital asset that needs to be managed properly to ensure its preservation (Muniz 2013). The loss of such knowledge can have a huge impact on the economy of a country. It is therefore of great importance that knowledge - more specifically tacit knowledge - is managed in forms that enhance its preservation and sharing.

\section{Knowledge management process models}

Knowledge sharing forms a cornerstone of many organisations' knowledge management strategy. Knowledge sharing practices and initiatives often form a key component of knowledge management programmes, in terms of organisational or individual learning (Laudon \& Laudon 2016; Riege 2005). Various knowledge management models to facilitate sharing of knowledge exist. One of the most popular models is the SECI (Socialisation, Externalisation, Combination, Internalisation) cycle model proposed by Nonaka and Takeuchi (1995) (see Figure 2). Although the SECI model focuses on knowledge creation instead of sharing, the model can enhance the theoretical understanding of knowledge sharing. In fact, knowledge sharing is a precondition for creating new knowledge (Wallace 2007).

With reference to the SECI model, the process of conversation of tacit knowledge into a new tacit knowledge through social interaction and shared experiences takes place in the socialisation phase. The new tacit knowledge is usually acquired through activities such as observation and replication of practices of a more experienced person. Externalisation involves articulating tacit knowledge in the

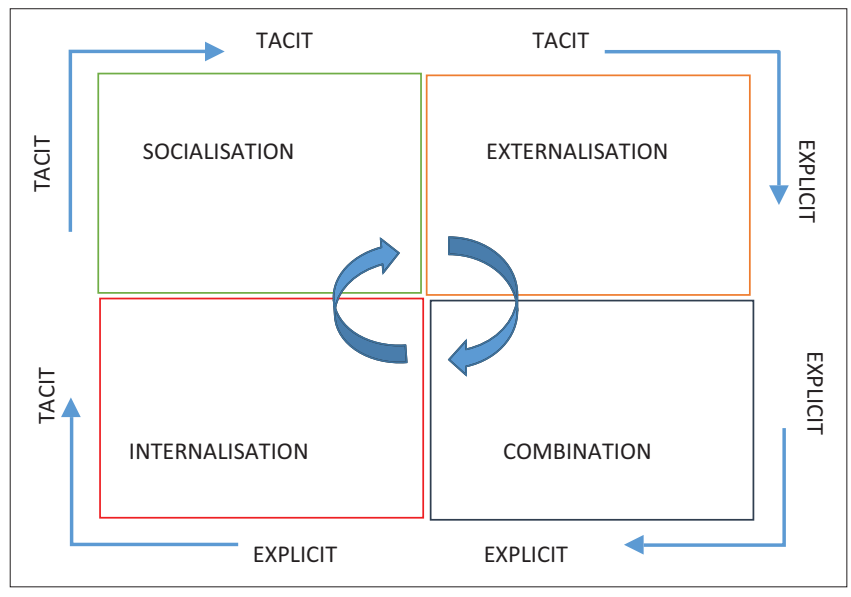

Source: Nonaka, I. \& Takeuchi, H., 1995, The knowledge-creating company: How Japanese companies create the dynamics of innovation, Oxford University Press, New York

FIGURE 2: The SECI model. form of explicit concepts such as metaphors, analogies, hypotheses and cognitive models by recombining different situations in order to create more explicit knowledge (Moreno \& Cavazotte 2015). In the combination phase, new explicit knowledge is created by merging, categorising and synthesising existing explicit knowledge (Wu et al. 2017). Internalisation involves converting explicit knowledge into a new tacit knowledge and is closely related to learning by doing (Alavi \& Leidner 1999; Wu et al. 2017). Although the SECI model is widely used, it has received some criticism most severe being the implicit assumption about cultural universality (Glisby \& Holden 2003). Another criticism of this model is that it is based on the Japanese culture embedded in Japanese companies' organisational behaviour (Bratianu 2010). A further reproach of the SECI model is that it lacks externalisation of tacit knowledge during online real-time chatting (Andreeva \& Ikhilchik 2011; Sjarbaini 2009; Wahlroos 2011). Applying the SECI model to this study for knowledge sharing is not appropriate, as Mozambique is culturally different from Japan. We apply the cognitive semiotic model proposed by Sjarbaini (2009) for knowledge sharing as a component of tacit knowledge is the cognitive element, which replicates mental models, beliefs and perceptions.

\section{The cognitive semiotic model}

Cognitive semiotics can be defined as an interdisciplinary matrix of disciplines and methods, focused on multifaceted phenomenon of meaning (Zlatev 2012). The cognitive side focuses more on the mental processes and structures. Among the methods used in the cognitive side for data gathering are prototype analysis, content analysis, artificial intelligence and computer simulation. The semiotic side is fundamentally based on the symbols and sign structure approach (Zlatev 2012). The combination of a cognitive approach and a semiotic approach to represent knowledge forms the foundation of the cognitive semiotic model. In the cognitive semiotic model, the process of acquiring knowledge is seen as a three-tier one, namely sensory knowledge, coded knowledge and theoretical knowledge.

The sensory knowledge is constructed on the first dimension of Charles Sanders Peirce semiotics, which relies on senses and is based on awareness (Purnama \& Susetyo-Salim 2017). There is no mediating sign associated with this knowledge and it is dependent on its context and relies on imitation (i.e. direct instruction and job training) as a mechanism for its transmission from one person to another. Hence, in the context of harvesting tacit knowledge from the traditional doctors we used video recordings and created computer animations to capture the sensory knowledge. For example, plant-harvesting mechanisms and ceremonial practices were captured by using a video camera.

The second tier in the cognitive semiotic model is the coded knowledge. This dimension introduces the use of codes to which an object or an experience refers. The coded knowledge promotes the possibility of communicating knowledge without the presence of that to which the knowledge refers. 
It is knowledge at descriptive level, for example describing how to apply a certain plant-based portion to a patient suffering from high fever. In our application, the coded knowledge was described by using series of pictures, videos, computer animations and notes taken during the interview process.

The third tier of the cognitive semiotic model is the theoretical knowledge. It puts the knowledge in perspective and refers to knowing the essence of a concept to be able to relate one concept to another. Theoretical knowledge essentially provides structure to coded knowledge and seeks to eliminate ambiguity in the code (Purnama \& Susetyo-Salim 2017). The theoretical knowledge of the traditional doctors of Mozambique was harvested and stored as a document comparing the healing practices to other well-documented practices of Chinese and Indian Ayurvedic practices. We only created a few such comparative documents to demonstrate the concept.

In efforts related to harvesting tacit knowledge, Pfeiffer (2005) developed a model called Most Important Knowledge Expertise (MIKE). The purpose of the MIKE model was to help researchers to accomplish their research objectives quicker by interrogating knowledge, information and workflow patterns within the available digital media. The authors developed a framework capable of extracting hidden knowledge by making use of network sensors and semantic web. The application model was subsequently subjected to interaction with domain experts. The experts were required to assist in the identification of the knowledge harvested, and to ensure accuracy and usefulness for the model. Our application, proposed in this article, makes use of domain expert both for requirement elucidation and for the usability testing. Similarly, Mbananga (2012) proposed a knowledge harvesting application based on a model they called Systematic Knowledge Harvesting Architecture Model (SKHAM). The SKHAM model was based on a case study conducted in South Africa. The focus of this study was to establish an institutional memory, support sharing of knowledge, learn from previous lessons and enforce the culture of learning organisation. SKHAM was designed to identify knowledge needs, based on problem identification, interaction, processes, knowledge structure and quality assurance. Mbananga (2012) concluded that the process of harvesting knowledge requires willingness, passion and faithfulness from the employees. Brown and Kruger (2010) proposed an architecture to combine the benefits of the Knowledge Harvesting System and Business Process Management. Their model was designed to provide a foundation for research and development of a processoriented knowledge management system. In their research, domain experts were used to verify whether knowledge has been captured or not. Their results showed that the model could enable designers of a repository to choose between making use of an existing template or designing a new one.

\section{Research methodology}

To achieve our research objectives, data from 13 randomly selected traditional doctors situated in rural areas around the capital city Maputo were collected. Most of the participants (77\%) were herbalists and the remaining 23\% were diviners. Among the participants, $15.38 \%$ had completed secondary school education, and the remaining $84.62 \%$ had only acquired some levels of primary education. Most of the participants were female $(69.23 \%)$. The ages of the participants ranged from 30 to 70 years and each had more than 6 years of experience. Communication among the participants concerning the acquisition and sharing of knowledge happened mostly during informal social gatherings and formal meetings organised by AMETRAMO.

Both qualitative and quantitative data were collected by using semi-structured interviews, questionnaires and observations. The interview questions were based on a combination of knowledge management processes found in the literature, and interviews were conducted at the traditional doctors' premises. Procedures and practices applied in the healing process of various ailments, plants used, methods of preparation and administering of the medication were captured by using photographs, video and voice recordings. These data were then used in the requirements elucidation and the design phases of the system development. For the purpose of usability testing, 10 of the 13 participants were used. Three of the participants could not be available for usability testing because of some prior commitments.

Based on the cognitive semiotic principles, we modelled the application by using the Unified Modelling Language model. The cloud architecture was used to promote the availability of the system. The software system was developed by using Java programming language. MySQL version 5.7 database was selected as a repository for storing the tacit knowledge captured in the forms of text, voice, videos, photographs and animations. XAMPP was chosen as the server to host the application. Java was used as it enables cross-platform deployment of the application, MySQL database was selected as it is an open source database management tool with support for security, reliability and scalability. The choice of XAMPP was based on the fact that it is a free, open source web server that is easy to install. The usability of the system was tested by using the heuristic evaluation method.

\section{System design and implementation}

The dual objective of our system was that it must be able to capture tacit knowledge and be able to support users with minimal computing skills. To achieve these objectives, we had extensive consultations with the traditional knowledge holders. Several methods and techniques such as interviews, questionnaires, video and audio recordings of the traditional practices were taken. These data were then used to elucidate the systems requirements and design of the knowledge repository. Based on the inputs received from the participants 


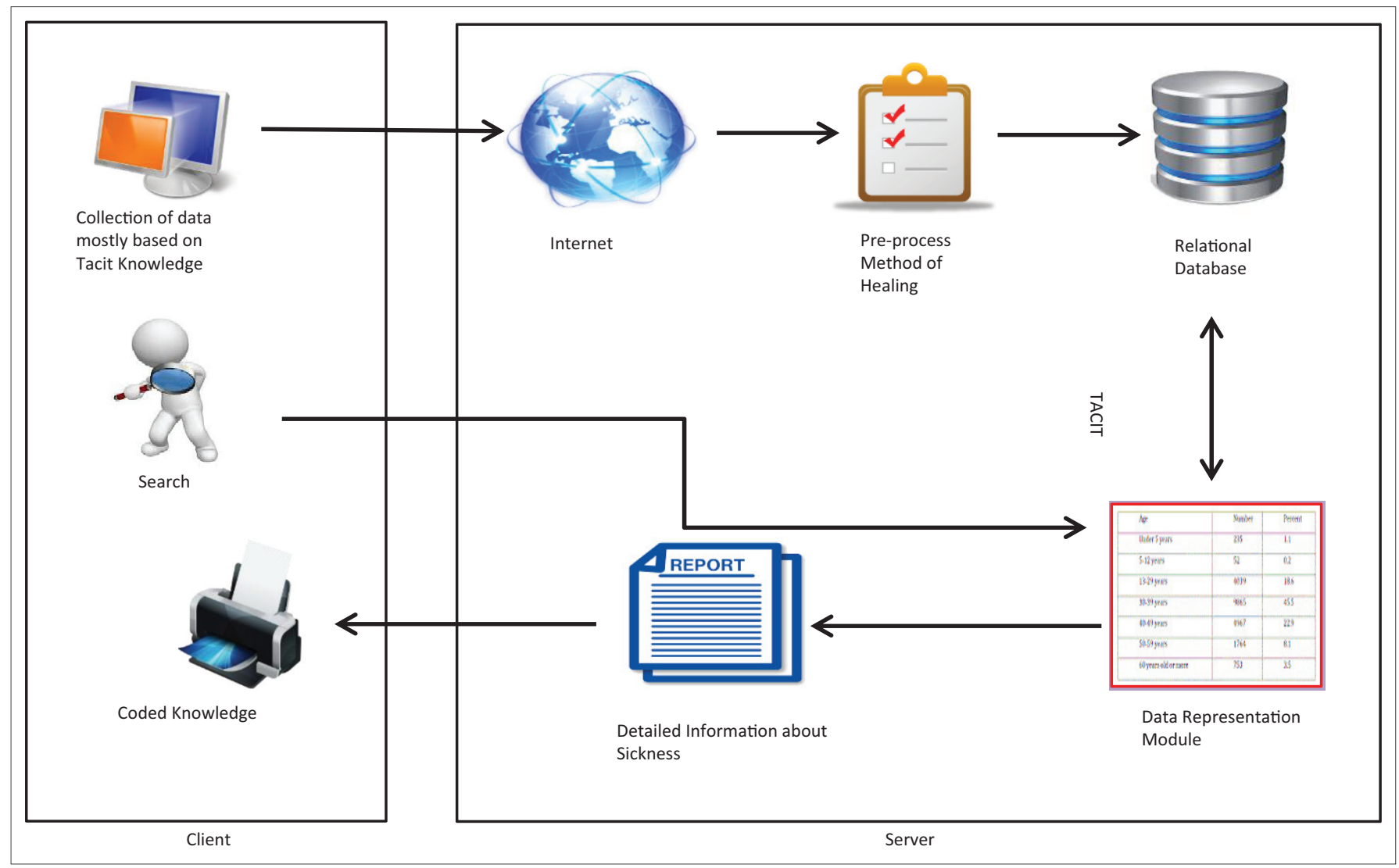

FIGURE 3: System architecture of the application.

a Unified Modelling Language model was designed. The model was then implemented in Java. Cloud architecture was used to promote availability of the system.

Based on the types of traditional health services, i.e. herbalists, diviners and prophets, the tacit knowledge associates in these healing practices are captured by using appropriate tools. Before this information is stored in the database, preprocessing activities are performed on it to assist with its categorisation. The categorisation assists in quick retrieval of information from the database (see Figure 3).

A module to assist with query or search the database and produce reports that are relatively easy to understand was incorporated into the system. For instance, the report (codified knowledge) incorporated both texts and pictures. If any videos are associated with the report, they are displayed on the client's screen. The report can be used for guiding other traditional doctors on how to treat a particular ailment or to compare and analyse various ways of treating a particular ailment.

\section{Results analysis and discussion}

The usability testing of the developed application is presented in this section.

\section{Usability testing}

There are numerous methods to evaluate a system's usability. In this study, usability testing was conducted by using the heuristic evaluation method, designed to assist in the identification of functionality and interface problems (Nielsen 1992, 1994). Participants involved in the testing of the application were given an evaluation template based on heuristic evaluation method, designed to assist in testing: visibility of the system status, match between system and the real world, user controls, consistency and standards of system, flexibility and efficiency of use and error prevention. To facilitate in testing these activities, the participants were required to perform certain common tasks. These tasks were performed on a laptop running Windows 8.1 and were conducted at the premises of the traditional doctors. While the participants were performing their assigned tasks, their activities were monitored closely to analyse the difficulties encountered in using the system. Table 1 shows the list of the tasks, number of incidences that occurred while performing the associated tasks and the percentages of participants that needed assistance in completing them.

From the data presented in Table 1, 90\% of the participants encountered problems while adding information related to illnesses, symptoms and their treatments. This was attributed to the fact that multiple text areas needed to be filled, and a number of widgets (e.g. radio buttons and combo boxes) needed to be selected before the information could be submitted to the database. A similar number (90\%) of participants had problems while performing activities related to generating reports and searching for information saved on the server. These activities required the participants to enter or select items of interest by scrolling or navigating to another 
TABLE 1: Tasks performed during usability testing

\begin{tabular}{|c|c|c|c|}
\hline Task & Tasks associated with & Number of incidences & $\begin{array}{l}\text { Participants needed } \\
\text { assistance }(\%)\end{array}$ \\
\hline \multirow[t]{4}{*}{ A: Registering themselves } & User could not find the menu item for registering themselves. & 2 & 60 \\
\hline & User could not correctly set their birthdate in the required format. & 6 & \\
\hline & User could not match the set password during the confirmation stage. & 0 & \\
\hline & User failed to set the mandatory fields. & 2 & \\
\hline \multirow[t]{3}{*}{ B: Login by using their credentials } & User could not find the menu item to login. & 0 & 20 \\
\hline & User typed incorrect information. & 2 & \\
\hline & User forgot pin or password. & 0 & \\
\hline \multirow{6}{*}{$\begin{array}{l}\text { C: Add information related to illnesses, } \\
\text { symptoms and treatments }\end{array}$} & User could not find on the menu to add a new sickness. & 2 & 90 \\
\hline & User failed to make use of combo boxes. & 5 & \\
\hline & User failed to make use of radio buttons. & 4 & \\
\hline & User failed to make use of check buttons. & 4 & \\
\hline & User failed to set the mandatory fields. & 6 & \\
\hline & User failed to add or delete items in the text boxes. & 7 & \\
\hline \multirow{3}{*}{ D: Generating reports } & User could not select a specific report to generate. & 7 & \\
\hline & User failed to view or print the report. & 8 & \\
\hline & User tried to generate a second report without closing the first report window. & 8 & \\
\hline \multirow[t]{2}{*}{ E: Performing search activities } & User failed to select the desired search items. & 5 & 90 \\
\hline & User could not migrate to the search window. & 5 & \\
\hline
\end{tabular}

screen. To minimise the number of incidences reported while performing activities related to these tasks, buttons for common searches and report creation were added. During the process of registering themselves, $60 \%$ of the participants needed help. Activity related to entering of the birthdate in the specified format was observed to be a little problematic. This problem was reduced by providing suitable formatted dropdown lists for the day, month and year.

In addition to performing the assigned tasks, the participants were also required to comment on aspects related to the heuristic evaluation categories: visibility of the system status, match between system and the real world, user controls, consistency and standards of system, flexibility and efficiency of use and error prevention. Figure 4 shows the number of usability issues identified in each of these categories. The visibility of system status requires that the system keep the users informed of the activities taking place within a reasonable period. To minimise issues identified in this category, our application made use of messages such as 'please wait, your query is being processed' during search operations and progress bars while authenticating a user.

A requirement to enhance usability of a system is that there should be a match between system and real world. This is to ensure that the users are presented with familiar terms and phrases. One of the reasons for the large number of usability issues arising in this category (see Figure 4) is because interface of our system displayed information in English, whereas the participants in this study are Portuguesespeaking with some understanding of English. To minimise this gap between our system and the Portuguese-speaking users, we made extensive use of tooltips, dialog boxes and pop-up windows written in Portuguese (see Figure 5).

Although most of the traditional doctors (70\%) had written down record-keeping in place, very few commonalities were observed in the details of these records and in the format in

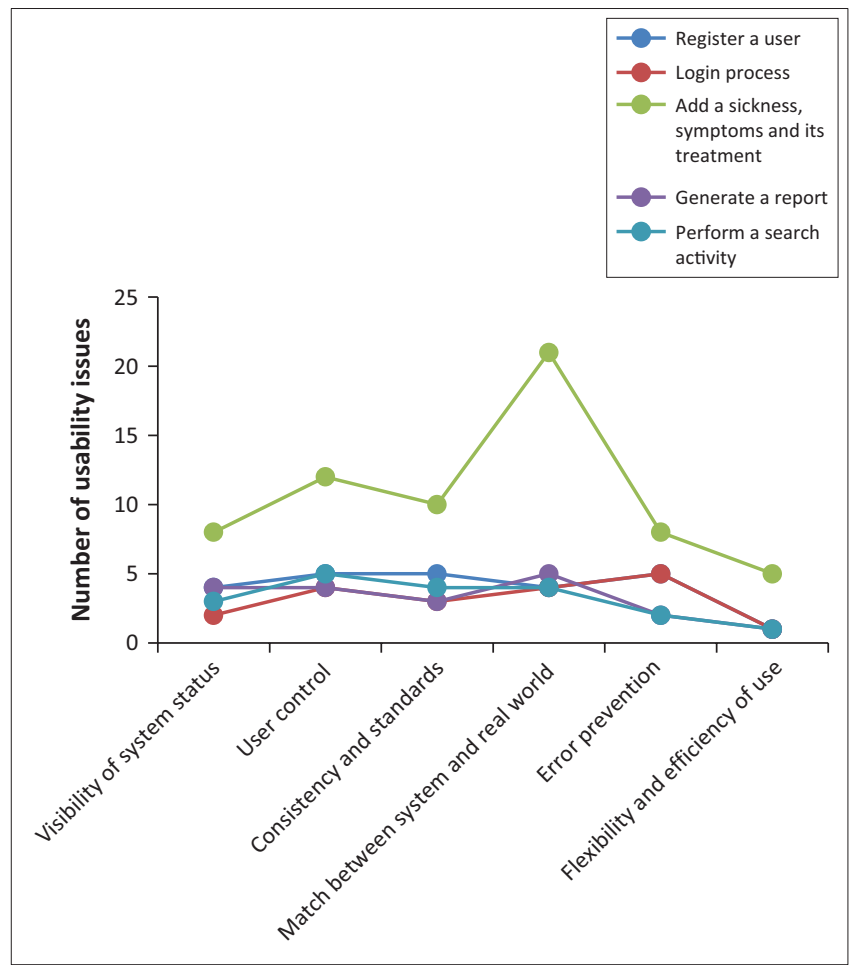

FIGURE 4: Number of usability issues identified in the various heuristic evaluation categories.

which they were kept. Hence, it was difficult to design the electronic forms in a way that matched their forms. This contributed to the large gap between the system and the users' world.

The Consistency and standards requires that the look-and-feel of the interface presented to a user be consistent and follow a set standard throughout the entire application. To reduce the number of inconsistencies identified while completing the tasks related to adding an ailment, its symptoms and treatment (see Figure 4), the look-and-feel of the widgets and their layout were redesigned. For example, the submit 


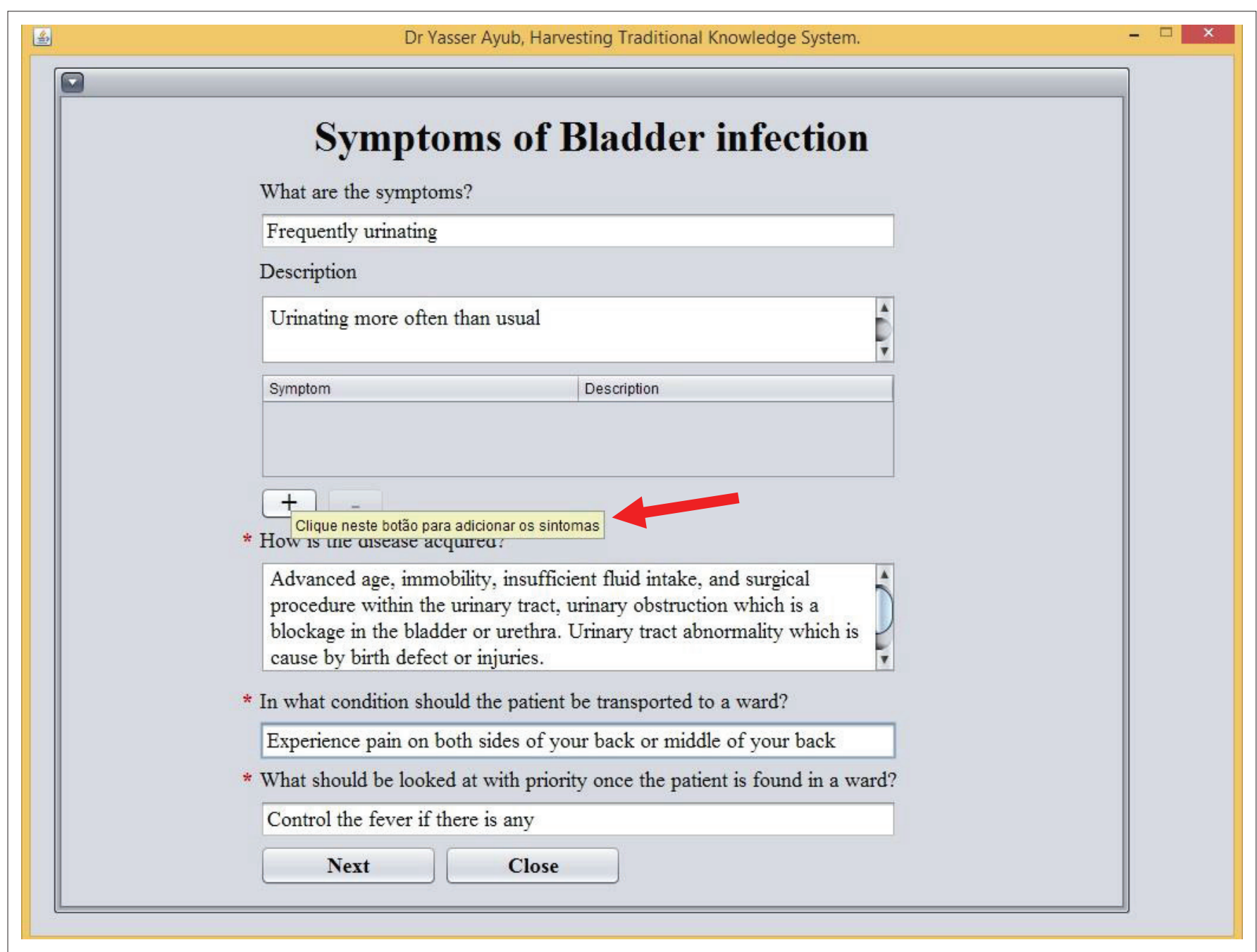

FIGURE 5: Input window using tooltip in Portuguese.

buttons for submitting a query and submitting personal details during registration look the same and perform similar actions. The principle of flexibility and efficiency of use requires that the system cater for both experienced and novice users. In line with this principle, our system made use of shortcut keys to minimise mouse actions. Realising that the users of our systems are not all computer literate and make many mistakes, our system incorporated features such as undo and redo and back buttons to navigate back to a previous screen. These features enhanced user control. Another important element of the heuristic evaluation is error prevention. The principle of error prevention is based on the fact that a system should be designed to eliminate error-prone conditions. To minimise the identified usability issues related to the principle of error prevention, our application added radio buttons, check boxes and combo boxes to reduce extensive typing.

\section{Ethical consideration}

This work was ethically approved by the Faculty of Information and Communication Technology committee on research ethics of Tshwane University of Technology, South Africa. Ref\#: FCRE/ICT/2015/04/007.

\section{Conclusion}

\section{Summary, conclusions and future work}

The traditional doctors of Mozambique have a rich heritage of medicinal plants usage and possess great knowledge typically in a tacit form. To avoid erosion of this indigenous knowledge, we presented a computer-based application to convert, capture and store tacit knowledge held by the traditional doctors for its preservation and sharing. Methods such as interviews, questionnaires, observations, photographs, video and voice recordings and computer animations were used in converting tacit knowledge into an explicit form. We observed that the cognitive semiotic model not only assisted in capturing the tacit knowledge but also had an influence on the design of the computer application. The developed application was subjected to heuristic evaluation for its usability. The overall test participants' response to the application was positive. However, a major concern identified during the usability testing was the match between system and real world. A contributing factor was the difference in the language in which the users are conversant (Portuguese) and the language used on the application interface (English). This gap was minimised by providing tooltips in Portuguese. It was observed that the developed 
application could support users with little formal education. However, some basic computer literacy training would assist in maximising the benefits of the system. In this regard, organisations such as AMETRAMO could play a central role.

As an extension to this work, we aim to increase the use of Portuguese in the look-and-feel of the user interface, and in the reports generated. This will simplify the activities related to knowledge capturing and improve on the general acceptance of the system. We also aim at providing voice dictation capabilities to enhance the usability of the system.

\section{Acknowledgements}

The authors would like to thank Tshwane University of Technology for its financial support and the faculty of ICT committee on research ethics for approving this research (Ref\#: FCRE/ICT/2015/04/007).

\section{Competing interests}

The authors declare that they have no financial or personal relationships that may have inappropriately influenced them in writing this article.

\section{Authors' contributions}

Y.I.R.A. was involved in data collection and implementation of the software system. O.P.K. was involved in the design of the software system, heuristic testing and in the writing of the article. M.L. was involved in the heuristic testing, writing of the article and its proofreading.

\section{References}

Alavi, M. \& Leidner, D.E., 1999, 'Knowledge management systems: Issues, challenges, and benefits', Communications of the AIS 1(7), 1-37.

Andreeva, T. \& Ikhilchik, I., 2011, 'Applicability of the SECI model of knowledge creation in Russian cultural context: Theoretical analysis', Knowledge and Process Management 18, 56-66. https://doi.org/10.1002/kpm.351

Bratianu, C., 2010, 'A critical analysis of the Nonaka's model of knowledge dynamics', in Proceedings of the 2nd European Conference on Intellectual Capital, 2010, Academic Publishing Limited, Reading, March 29-30, pp. 115-120.

Bratianu, C.A. \& Orzea, I., 2010, 'Tacit knowledge sharing in organizational knowledge dynamics', in Proceedings of the 2nd European Conference on Intellectual Capital, 2010, Lisbon, Portugal, March 29-30, pp. 107-1114.

Brown, M.L. \& Kruger, M.J., 2010, 'A net-centric approach to tacit knowledge management', in eChallenges, 2010, IEEE, Warsaw, Poland, October 27-29, pp. 1-8.

Bruschi, P., Morganti, M., Mancini, M. \& Signorini, M.A., 2011, 'Traditional healers and laypeople: A qualitative and quantitative approach to local knowledge on medicinal plants in Muda (Mozambique)', Journal of Ethnopharmacology 138, 543-563. https://doi.org/10.1016/j.jep.2011.09.055

Burnett, S., Macafee, C. \& Williams, D., 2017, 'Applying a knowledge conversion mode to cultural history: Folk song from oral tradition to digital transformation', Electronic Journal of Knowledge Management 15(2), 61-71.

Geisler, E. \& Wickramasinghe, N., 2015, Principles of knowledge management: Theory practice, and cases, Routledge, New York.

Gibert-Tisseuil, F., 1997, 'Reflections on traditional Chinese medicine and its pharmacopoeia', Annales Pharmaceutiques Francaises 56, 282-285.

Glisby, M. \& Holden, N., 2003, 'Contextual constraints in knowledge management theory: The cultural embeddedness of Nonaka's knowledge-creating company' Knowledge and Process Management 10, 29-36. https://doi.org/10.1002/ kpm.158

Googlemaps, 2016, Map of southern Africa, viewed 23 April 2016, from https://www. google.co.za/maps/@-20.0347085,27.132872,4.65z?hl=en
Grover, R. \& Froese, T.M., 2016, 'Knowledge management in construction using a SocioBIM platform: A case study of AYO smart home project', Procedia Engineering 145, 1283-1290. https://doi.org/10.1016/j.proeng.2016.04.165

Hey, J., 2004, 'The data, information, knowledge, wisdom chain: The metaphorical link', Intergovernmental Oceanographic Commission 26, 1-18.

Howells, J.R. 2002. 'Tacit knowledge, innovation and economic geography', Urban Studies 39, 871-884. https://doi.org/10.1080/00420980220128354

Johannessen, J.-A., Olaisen, J. \& Olsen, B., 2001, 'Mismanagement of tacit knowledge: The importance of tacit knowledge, the danger of information technology, and what to do about it', International Journal of Information Management 21, 3-20. https://doi.org/10.1016/S0268-4012(00)00047-5

Laudon, K.C. \& Laudon, J.P., 2016, Management information system, Pearson Education India, Chennai, India.

Lechner, E., 2009, 'Migração, saúde e diversidade cultural', Lisboa: Imprensa de Ciências Sociais 85, 85-119.

Mbananga, N., 2012, 'Knowledge harvesting: An experiential case study', in 2012 Abudhabi 3rd Knowledge Management Conference in Abudhabi, Centre for Informatics, Knowledge Management and Knowledge Economy, Abu Dhabi, October 23-25, pp. 1-13.

Misau, 2004, 'Política da Medicina Tradicional e Estratégia da sua implementação', in M.D. Saúde (ed.), Plano estratégico nacional de prevenção e controlo das doenças não transmissiveis, pp. 1-51, Ministério da Saúde, Mozambique.

Moreno, V. \& Cavazotte, F., 2015, 'Using information systems to leverage knowledge management processes: The role of work context, job characteristics and tasktechnology fit', Procedia Computer Science 55, 360-369. https://doi.org/10.1016/ j.procs.2015.07.066

Muniz, A.E., 2013, The retention of tacit knowledge in higher learning administration, ProQuest LLC Publishers, Ann Arbor, MI.

Neches, R., Fikes, R.E., Finin, T., Gruber, T., Patil, R., Senator, T. \& Swartout, W., 1991 'Enabling technology for knowledge sharing', Al Magazine 12(2), 36-56.

Nielsen, J., 1992, 'Finding usability problems through heuristic evaluation', in Proceedings of the SIGCHI Conference on Human Factors in Computing Systems, ACM, Monterey, CA, May 2-7, pp. 373-380.

Nielsen, J., 1994, Usability engineering, Morgan Kaufmann, San Francisco, CA.

Nonaka, I., 2008, The knowledge-creating company, Harvard Business Review Press, Boston, MA.

Nonaka, I. \& Takeuchi, H., 1995, The knowledge-creating company: How Japanese companies create the dynamics of innovation, Oxford University Press, New York.

Pfeiffer, J., 2005, 'Commodity fetichismo The Holy Spirit, and the turn to Pentecostal and African independent churches in Central Mozambique', Culture, Medicine and Psychiatry 29, 255-283. https://doi.org/10.1007/s11013-005-9168-3

Pfeiffer, J., Gimbel-Sherr, K. \& Augusto, O.J., 2007, 'The holy spirit in the household Pentecostalism, gender, and neoliberalism in Mozambique', American Anthropologist 109, 688-700. https://doi.org/10.1525/aa.2007.109.4.688

Polanyi, M., 1966, The tacit dimension, Peter Smith, Gloucester, MA.

Polanyi, M., 1983, The tacit dimension, Peter Smith, Gloucester, MA, pp. 4-49, originally published 1966 .

Polanyi, M., 2015, Personal knowledge: Towards a post-critical philosophy, University of Chicago Press, Chicago, IL.

Presbitero, A., Roxas, B. \& Chadee, D., 2017, 'Sustaining innovation of information technology service providers: Focus on the role of organisational collectivism', International Journal of Physical Distribution \& Logistics Management 47, 156174. https://doi.org/10.1108/IJPDLM-07-2015-0171

Purnama, R. \& Susetyo-Salim, T.A., 2017, 'Using cognitive-semiotic model for capturing tacit knowledge: Case study of Indonesian traditional massage', Medwell Journals 2, 15-19.

Riege, A., 2005, 'Three-dozen knowledge-sharing barriers managers must consider', Journal of Knowledge Management 9, 18-35. https://doi.org/10.1108/ 13673270510602746

Sjarbaini, V.L.R.N., 2009, The knowledge dynamics of organizational innovation Understanding the implementation of decision support for planners, PrintPartners Ipskamp B.V., Enschede, Netherlands.

Soto-Acosta, P., Popa, S. \& Palacios-Marqués, D., 2017, 'Social web knowledge sharing and innovation performance in knowledge-intensive manufacturing SMEs', The Journal of Technology Transfer 42, 425-440. https://doi.org/10.1007/s10961 016-9498-z

Wahlroos, J.K., 2011, Social media as a form of organizational knowledge sharing, Helsingfors Universitet, Helsinki, Finland.

Walker, A.M., 2017, 'Tacit knowledge', European Journal of Epidemiology 32(4), $261-$ 267. https://doi.org/10.1007/s10654-017-0256-9

Wallace, D.P., 2007, Knowledge management. Historical and cross-disciplinary themes, Libraries Unlimited, Westport, CT.

WHO, 2004, World health report. Changing history, WHO, Geneva, Switzerland.

Wu, Y.-W., Liao, S., Wen, M.-H. \& Weng, K.-H., 2017, 'A knowledge conversion model based on the cognitive load theory for architectural design education', Eurasia Journal of Mathematics, Science \& Technology Education 13, 2367-2385.

Zlatev, J., 2012, 'Cognitive semiotics: An emerging field for the transdisciplinary study of meaning', Public Journal of Semiotics 4, 2-24. 